\title{
EFICÁCIA DE ISOXAFLUTOLE NO CONTROLE DE PLANTAS DANINHAS NA CULTURA DA BATATA
}

\author{
Márcio L. Adoryan ${ }^{1}$, Maria do Carmo S.S. Novo ${ }^{2}$, Patrícia Favoretto ${ }^{3}$, \\ João Tessariolli Neto ${ }^{4}$ e Paulo César T. Melo ${ }^{4}$
}

${ }^{1} E_{\text {Eng }}$. Agrônomo, Bayer CropScience. Caixa Postal 921. Paulínia, SP 13140-000 marcio.adoryan@bayercropscience.com

${ }^{2} E_{n}{ }^{a}$. Agrônoma MSc., Pesquisadora Científica, Centro de Ecofisiologia e Biofísica, Instituto Agronômico (IAC). Campinas, SP

${ }^{3}$ Eng $^{\mathrm{a}}$. Agrônoma Mestranda em Fitotecnia. USP/ESALQ. Piracicaba, SP 13418-900

${ }^{4}$ Professor. Departamento de Produção Vegetal, USP/ESALQ. Piracicaba, SP

\section{RESUMO}

O experimento foi conduzido durante o ano agrícola de 2002, na Estação Agrícola Experimental da Bayer CropScience, localizada em Paulínia, SP, em solo argiloso cultivado com batata cv. Monalisa. Os objetivos do experimento foram avaliar a eficácia de isoxaflutole no controle de espécies daninhas, verificar sua seletividade à cultura de batata e seu efeito na produção de tubérculos. O delineamento experimental utilizado foi em blocos ao acaso com sete tratamentos e quatro repetições. As parcelas experimentais foram compostas de quatro linhas de $7 \mathrm{~m}$ de comprimento, espaçadas em $0,60 \mathrm{~m}$. Os tratamentos foram: testemunhas com e sem capina, as doses de isoxaflutole $(0,075,0,150$ e $0,300 \mathrm{~kg} / \mathrm{ha})$ e os padrões metribuzin $(0,720 \mathrm{~kg} / \mathrm{ha}) \mathrm{e}$ linuron + metribuzin $(0,910+0,384 \mathrm{~kg} / \mathrm{ha})$. As avaliações da percentagem de controle das espécies daninhas e de seletividade à cultura foram realizadas aos 14, 28 e 42 dias após os tratamentos empregando-se uma escala visual na qual zero significa nenhum controle ou nenhum dano à cultura e $100 \%$ correspondeu ao controle total da planta daninha ou morte da planta da cultura. As principais espécies presentes na área experimental foram: Digitaria horizontalis Willd., Brachiaria plantaginea (Link.) Hitch, Galinsoga parviflora Cav., Raphanus raphanistrum L. e Amaranthus viridis L.. Observou-se que isoxaflutole foi seletivo à cultura da batata. As espécies daninhas presentes foram eficientemente controladas com isoxaflutole não havendo diferença do tratamento com metribuzin. Os tratamentos com isoxaflutole foram mais eficientes do que aquele com metribuzin + linuron no controle das gramíneas. Isoxaflutole não afetou a produção de tubérculos.

Palavras-chave: pré-emergência, herbicida, controle químico, isoxazole, Solanum tuberosum L.

\section{ABSTRACT \\ Efficacy of isoxaflutole in weed control in potato crop}

This experiment was carried out at Bayer CropScience Experimental Station, in Paulínia, SP, Brazil, in a loamy soil cultivated with potato cv. Monalisa, during the 2002 growing season. The objectives were to evaluate the efficacy of isoxaflutole in weed control, to verify its selectivity to the potato crop and its effect on tubercule yield. The experimental design was a completely randomized block with seven treatments and four replications. The experimental plots consisted of four $7 \mathrm{~m}$ rows, with plants spaced in $0.60 \mathrm{~m}$. The treatments were: controls (weedy and weedfree) and isoxaflutole rates $(0.075,0.150$ and 0.300 $\mathrm{kg} / \mathrm{ha})$. Metribuzin $(0.720 \mathrm{~kg} / \mathrm{ha})$ and linuron + metribuzin $(0.910+0.384 \mathrm{~kg} / \mathrm{ha})$ were used as standards. The evaluations of the percentage of weed control and the selectivity on the potato crop were carried out at 14, 28 and 42 days after treatment adopting a scale in which zero meant no weed control or no crop damage and one hundred corresponded to total control or death of the crop. The main weed species presented in the area were: Digitaria horizontalis Willd., Brachiaria plantaginea (Link.) Hitch, Galinsoga parviflora Cav., Raphanus raphanistrum L. and Amaranthus viridis L.. Potato plants did not show any visual symptoms of damage attributed to herbicide treatment. The species weed were efficiently controlled with isoxaflutole but there was no significant difference when compared with metribuzin. The treatments with isoxaflutole provided better control than linuron + metribuzin on grasses. Isoxaflutole did not affect the tubercule yield.

Key words: chemical control, isoxazole, preemergence application, Solanum tuberosum L. 


\section{INTRODUÇÃO}

A batata ocupa o quarto lugar entre os alimentos mais consumidos no mundo, sendo superada apenas pelo trigo, arroz e milho (Grando, 2002). A área cultivada no Brasil no ano de 1999 foi de, aproximadamente, 180 mil hectares, com volume de produção de 2,561 milhões de toneladas e produtividade média de 17 tha (Vieira, 2000). Esta produtividade pode ser considerada baixa quando comparada à de outros países e além disso, está muito aquém do que poderia ser conseguida no Brasil, onde lavouras que empregam tecnologia têm obtido mais de 30 t/ha de tubérculos e melhor qualidade final do produto.

Dentre as técnicas envolvidas no sistema de produção, o manejo adequado da população de plantas daninhas assume significativa importância por interferir diretamente no desenvolvimento da cultura. Em geral, é na primeira metade do ciclo vegetativo que ocorre maior competição entre as plantas daninhas e a cultura, podendo reduzir drasticamente a produção. É necessário manter a cultura no limpo nos primeiros 30 a 50 dias, época em que se faz a amontoa (Vieira, 2000). Em áreas com alta infestação de plantas daninhas, ocorre interferência mesmo depois da amontoa, justificando adotar métodos eficientes de controle para garantir uma boa produção. Em regiões onde o cultivo é mais tecnificado, é comum o uso de herbicidas em pré-plantio ou em pré-emergência, seguido de um ou dois cultivos (tração motorizada), os quais, geralmente, coincidem com a amontoa. Os herbicidas devem ser escolhidos em função de sua eficiência, segurança e economia, considerando o programa de rotação de culturas e outras recomendações técnicas para o cultivo (Vieira, 2000). Entretanto, a quantidade de herbicidas disponíveis para a cultura da batata ainda é pequena, embora tenha havido alguns acréscimos ultimamente (Gelmini \& Christoffoleti, 1997, Rodrigues \& Almeida, 1998 e Andrei, 1999).

O herbicida isoxaflutole pertence ao grupo químico dos isoxazoles, cuja absorção se verifica principalmente através do meristema apical das plântulas, raízes e colo das plantas, onde, via xilema, se transloca para as folhas (Rodrigues \& Almeida, 1998). Seu mecanismo de ação está relacionado com o impedimento da biossíntese de pigmentos carotenóides, essenciais para a proteção da clorofila, através da inibição da enzima 4-hydroxyphenyl-pyruvate-dioxygenase. Os carotenos direcionam a energia luminosa às moléculas de clorofila. Também ajudam a dissipar o excesso de energia das moléculas de clorofila após a absorção da energia luminosa. Se os carotenos estão ausentes, não há como dissipar o excesso de energia das moléculas de clorofila, o que pode causar danos às membranas das plantas. As moléculas de clorofila podem também ser danificadas devido a esse excesso de energia através de um processo conhecido como foto-oxidação (Hager \& McGlamery, 1999). Sem clorofila, o processo de fotossíntese é inibido causando a morte da planta (Prostko \& Baugham, 1999). Como resultado, as partes afetadas das plantas tornam-se de brancas a translúcidas (Gunsolus \& Curran, 1999). Os sintomas iniciam-se no ápice e nas margens das folhas novas (Regehr, 1998) e são mais evidentes nos períodos de maior pluviosidade em solos mais leves e pobres em matéria orgânica (Rodrigues \& Almeida, 1998).

Plantas tolerantes à isoxaflutole podem metabolizar rapidamente o ingrediente ativo a uma forma não tóxica, como já foi observado na cultura do milho (Gibson, 2001). Fatores que atrasam o desenvolvimento e o crescimento da planta, tais como baixa temperatura e umidade excessiva podem causar injúria à cultura e retardar a metabolização do herbicida causando fitotoxicidade (Wicks et al., 2002; Hager \& McGlamery, 1999 e Gibson, 2001.

No solo, esse herbicida é adsorvido pelos colóides e apresenta baixa mobilidade, exceto em solos arenosos ou com baixo teor de matéria orgânica (Rodrigues \& Almeida, 1998 e Mitra et al., 1999). Sua principal fonte de degradação é a microbiana, e em condições adversas ocorre também a decomposição química. As perdas por fotodecomposição e/ou volatilização são insignificantes (Rodrigues \& Almeida, 1998).

Com a finalidade de aumentar o número de opções para o controle de plantas daninhas em batata, procurou-se avaliar a eficácia do herbicida isoxaflutole aplicado em pré-emergência em solo argiloso, sua seletividade à cultura e seu efeito na produção de tubérculos.

\section{MATERIAL E MÉTODOS}

O experimento foi instalado na Estação Agrícola Experimental da Bayer CropScience, localizada no município de Paulínia, SP, em solo argiloso cuja a análise de fertilidade se encontra na Tabela 1 . O solo foi corrigido e adubado de acordo com as instruções de Miranda Filho (1996).

O plantio dos tubérculos foi realizado em 03/04/02 utilizando-se o cultivar Monalisa. As parcelas foram compostas de quatro linhas de $7 \mathrm{~m}$ de comprimento espaçadas em $0,60 \mathrm{~m}$ estando os tubérculos-sementes distantes entre si em 0,35 m.

O experimento foi disposto em blocos ao acaso, com sete tratamentos e quatro repetições. Os tratamentos foram os seguintes: testemunha capinada, testemunha com plantas daninhas, isoxaflutole nas doses de $0,075,0,150$ e $0,300 \mathrm{~kg} / \mathrm{ha} \mathrm{e}$ os padrões metribuzin $(0,720 \mathrm{~kg} / \mathrm{ha})$ e linuron + metribuzin $(0,910$ $+0,384 \mathrm{~kg} / \mathrm{ha}$ ). Os herbicidas foram aplicados em pré-emergência, com pulverizador costal de ar comprimido, dotado de barra com seis bicos de jato plano da série XR 110.015, com consumo de calda correspondente a $200 \mathrm{~L} / \mathrm{ha}$. As condições climáticas no momento da aplicação e durante o desenvolvimento da cultura foram adequadas para o desenvolvimento da cultura. Para manter a umidade do solo adequada, sempre que necessário foram realizadas irrigações por aspersão na área experimental.

As avaliações da eficiência dos herbicidas no controle de plantas daninhas e da seletividade à cultura foram realiza- 
das nas duas linhas centrais aos 14, 28 e 42 dias após o tratamento (DAA), por amostragem, em área correspondente a 5\% de cada parcela. Nessas ocasiões, efetuaram-se observações visuais do controle geral pelo método da percentagem de infestação (0 a $100 \%$ ) e aparecimento de fitotoxicidade nas plantas de batata pela ação dos herbicidas, onde zero significava nenhum controle de plantas daninhas ou nenhum dano à cultura e $100 \%$ correspondeu ao controle total da planta daninha ou morte da planta da cultura. Considerou-se como eficiente o herbicida que apresentou controle igual ou maior que $80 \%$ para cada espécie de planta daninha. A produção dos tubérculos foi avaliada nas duas linhas centrais das parcelas.

Os resultados obtidos foram submetidos à análise de variância e, quando significativas, as médias foram comparadas pelo teste de Tukey a 5\% de probabilidade. Para análise de variância, os valores expressos em percentagem foram transformados em arco seno raiz de $\mathrm{x} / 100$.

\section{RESULTADOS E DISCUSSÃO}

Em relação à fitotoxicidade, verificou-se que isoxaflutole, em todas as doses aplicadas, à semelhança dos padrões, foi seletivo à cultura, não tendo sido observado sintomas de injúria aos $42 \mathrm{DAA}$ (Tabela 2). O mesmo foi observado por Adoryan et al. (2002), na cultura de batata, onde foram aplicadas as doses de 0,075 e 0,1125 kg/ha.

As principais espécies presentes na área experimental foram: Digitaria horizontalis Willd. (capim-colchão), Brachiaria plantaginea (Link.) Hitch (capim-marmelada), Galinsoga parviflora Cav. (picão-branco), Raphanus raphanistrum L. (nabiça) e Amaranthus viridis L. (caruru-damancha).
Verificou-se que os níveis de controle dos capins marmelada e colchão foram elevados desde a amostragem realizada aos 14 DAA, mantendo-se no mesmo patamar até os 42 DAA (Tabela 3).

Para o capim-marmelada, aos 14 DAA, apenas a mistura de metribuzin + linuron apresentou controle inferior a $90 \%$, entretanto, não diferiu do tratamento com metribuzin (Tabela 3). As parcelas tratadas com isoxaflutole não diferiram entre si e apresentaram controle superior àqueles obtidos nos demais tratamentos. Aos $28 \mathrm{DAA}$, os tratamentos com isoxaflutole mostraram controles superiores aos obtidos nos tratamento com metribuzin + linuron. Para a dose de $0,075 \mathrm{~kg} / \mathrm{ha}$ este foi estatísticamente igual àquele obtido pelo metribuzin. Resultados semelhantes foram observados na avaliação realizada aos 42 DAA por Spader \& Vidal (2000) na cultura do milho, onde isoxaflutole controlou com eficiência o capim-marmelada até os 55 DAA.

O capim-colchão foi controlado com eficiência pelas doses de isoxaflutole e pelo metribuzin (Tabela 3). Aos 14 dias, o controle com as diferentes doses isoxaflutole foi superior ao dos padrões mas, aos $28 \mathrm{DAA}$, não diferiram do tratamento com metribuzin. Aos 42 DAA, as doses de 0,150 e 0,300 $\mathrm{kg} / \mathrm{ha}$ de isoxaflutole apresentaram controles superiores aos obtidos pelos padrões. Entretanto, a dose de 0,075 kg/ha embora tenha apresentado $97 \%$ de controle do capim-colchão não diferiu do tratamento com metribuzin. Young \& Hart (1998) e Adoryn et al. (2002) também observaram elevado controle de gramíneas anuais quando o isoxaflutole foi aplicado em pré-emergência.

Quanto à nabiça, verificou-se que, exceto aos $14 \mathrm{DAA}$, quando a dose menor de isoxaflutole apresentou $88 \%$ de controle, todos os outros tratamentos propiciaram excelente controle (Tabela 4). Entretanto, aos 28 DAA, a menor dose pro-

Tabela 1. Análise de fertilidade do solo de amostras coletadas no local do experimento. Paulínia, SP.

\begin{tabular}{|c|c|c|c|c|c|c|c|c|c|}
\hline $\mathbf{P}$ & M.O. & $\mathbf{p H}_{\mathrm{CaC1} 2}$ & $\mathbf{K}$ & $\mathrm{Ca}$ & Mg & $\mathbf{H}+\mathbf{A l}$ & SB & CTC & $\mathbf{V}$ \\
\hline mg.dm ${ }^{-3}$ & g.dm ${ }^{-3}$ & & & & & $\mathrm{dm}^{-3}$ & & & $\%$ \\
\hline 162 & 26 & 4,4 & 6,2 & 18 & 3 & 58 & 27,2 & 85,2 & 32 \\
\hline
\end{tabular}

Tabela 2. Seletividade à cultura, expressa em percentagem, nas avaliações realizadas aos 14, 28 e 42 dias após a aplicação. Paulínia, SP.

\begin{tabular}{lcccc}
\hline & $\begin{array}{c}\text { Dose } \\
\text { Tratamento }\end{array}$ & \multicolumn{2}{c}{ Percentagem de toxicidade à cultura $^{1}$} \\
Test. sem capina & 0 & 14 DAA & 28 DAA $\mathbf{~}^{\mathbf{2}}$ & 42 DAA \\
Test. capinada & 0 & $0 \mathrm{a}^{3}$ & $0 \mathrm{a}$ & $0 \mathrm{a}$ \\
Isoxaflutole & 0,075 & $0 \mathrm{a}$ & $0 \mathrm{a}$ & $0 \mathrm{a}$ \\
Isoxaflutole & 0,150 & $0 \mathrm{a}$ & $0 \mathrm{a}$ & $0 \mathrm{a}$ \\
Isoxaflutole & 0,300 & $0 \mathrm{a}$ & $0 \mathrm{a}$ & $0 \mathrm{a}$ \\
Metribuzin & 0,720 & $0 \mathrm{a}$ & $13 \mathrm{~b}$ & $0 \mathrm{a}$ \\
Metribuzin + linuron & $0,910+0,384$ & $0 \mathrm{a}$ & $11 \mathrm{~b}$ & $0 \mathrm{a}$ \\
\hline
\end{tabular}

1 Para análise, os dados foram transformados em arco seno de raiz de $\mathrm{x} / 100$.

2. DAA= dias após a aplicação dos produtos.

3. Médias seguidas pela mesma letra na coluna não diferem entre si pelo teste de Tukey a 5\% de probabilidade. 
Márcio L. Adoryan et al.

Tabela 3. Percentagem de controle dos capins marmelada (Brachiaria plantaginea (Link.) Hitch - BRAPL) e colchão (Digitaria horizontalis Willd. -DIGHO) nas avaliações realizadas aos 14, 28 e 42 dias após a aplicação. Paulínia, SP.

\begin{tabular}{|c|c|c|c|c|c|c|c|}
\hline \multirow[t]{3}{*}{ Tratamento } & \multirow{3}{*}{$\begin{array}{c}\text { Dose } \\
\text { (kg/ha) }\end{array}$} & \multicolumn{6}{|c|}{ Percentagem de controle $^{1}$} \\
\hline & & \multicolumn{3}{|c|}{ BRAPL } & \multicolumn{3}{|c|}{ DIGHO } \\
\hline & & $14 \mathrm{DAA}^{2}$ & 28DAA & 42DAA & 14 DAA & 28DAA & 42 DAA \\
\hline Test. sem capina & 0 & $0 c^{3}$ & $0 \mathrm{~d}$ & $0 \mathrm{~d}$ & $0 \mathrm{~d}$ & $0 \mathrm{c}$ & $0 \mathrm{~d}$ \\
\hline Test. capinada & 0 & $100 \mathrm{a}$ & $100 \mathrm{a}$ & $100 \mathrm{a}$ & $100 \mathrm{a}$ & $100 \mathrm{a}$ & $100 \mathrm{a}$ \\
\hline Isoxaflutole & 0,075 & $97 \mathrm{a}$ & $98 \mathrm{ab}$ & $93 \mathrm{ab}$ & $99 \mathrm{a}$ & $99 \mathrm{a}$ & $97 \mathrm{ab}$ \\
\hline Isoxaflutole & 0,150 & $100 \mathrm{a}$ & $100 \mathrm{a}$ & $100 \mathrm{a}$ & $100 \mathrm{a}$ & $100 \mathrm{a}$ & $100 \mathrm{a}$ \\
\hline Isoxaflutole & 0,300 & $100 \mathrm{a}$ & $100 \mathrm{a}$ & $100 \mathrm{a}$ & $100 \mathrm{a}$ & $100 \mathrm{a}$ & $100 a$ \\
\hline Metribuzin & 0,720 & $90 \mathrm{~b}$ & $93 \mathrm{~b}$ & $90 \mathrm{bc}$ & $93 \mathrm{~b}$ & $98 \mathrm{a}$ & $92 b$ \\
\hline Metribuzin + linuron & $0,910+0,384$ & $87 b$ & $87 c$ & $82 \mathrm{c}$ & $88 \mathrm{c}$ & $93 \mathrm{~b}$ & $86 c$ \\
\hline C.V. $(\%)$ & & 2,3 & 2,8 & 4,7 & 1,9 & 1,3 & 2,9 \\
\hline
\end{tabular}

1 Para análise, os dados foram transformados em arco seno de raiz de $\mathrm{x} / 100$.

2. $\mathrm{DAA}=$ dias após a aplicação dos produtos

3. Médias seguidas pela mesma letra na coluna não diferem entre si pelo teste de Tukey a 5\% de probabilidade.

Tabela 4. Percentagem de controle de nabiça (Raphanus raphanistrum L. - RAPRA) e de picão-branco (Galinsoga parviflora Cav. - GASPA) nas avaliações realizadas aos 14, 28 e 42 dias após a aplicação. Paulínia, SP.

\begin{tabular}{|c|c|c|c|c|c|c|c|}
\hline \multirow[t]{3}{*}{ Tratamento } & \multirow{3}{*}{$\begin{array}{l}\text { Dose } \\
\text { (kg/ha) }\end{array}$} & \multicolumn{6}{|c|}{ Percentagem de controle $^{1}$} \\
\hline & & \multicolumn{3}{|c|}{ RAPRA } & \multicolumn{3}{|c|}{ GASPA } \\
\hline & & $14 \mathrm{DAA}^{2}$ & 28 DAA & $\overline{42 \mathrm{DAA}}$ & 14 DAA & 28 DAA & 42 DAA \\
\hline Test. sem capina & 0 & $0 c^{3}$ & $0 \mathrm{c}$ & $0 \mathrm{~b}$ & $0 \mathrm{~b}$ & $0 \mathrm{~b}$ & $0 \mathrm{~b}$ \\
\hline Test. capinada & 0 & $100 \mathrm{a}$ & $100 \mathrm{a}$ & $100 \mathrm{a}$ & $100 \mathrm{a}$ & $100 \mathrm{a}$ & $100 \mathrm{a}$ \\
\hline Isoxaflutole & 0,075 & $88 \mathrm{~b}$ & $98 \mathrm{~b}$ & $96 a$ & $100 \mathrm{a}$ & $100 \mathrm{a}$ & $100 \mathrm{a}$ \\
\hline Isoxaflutole & 0,150 & $96 a$ & $100 \mathrm{a}$ & $100 \mathrm{a}$ & $100 \mathrm{a}$ & $100 \mathrm{a}$ & $100 \mathrm{a}$ \\
\hline Isoxaflutole & 0,300 & $100 \mathrm{a}$ & $100 \mathrm{a}$ & $100 \mathrm{a}$ & $100 \mathrm{a}$ & $100 \mathrm{a}$ & $100 \mathrm{a}$ \\
\hline Metribuzin & 0,720 & $96 a$ & $99 \mathrm{ab}$ & $99 \mathrm{a}$ & $100 \mathrm{a}$ & $100 \mathrm{a}$ & $100 \mathrm{a}$ \\
\hline Metribuzin + linuron & $0,910+0,384$ & $97 \mathrm{a}$ & $100 \mathrm{a}$ & $100 \mathrm{a}$ & $100 \mathrm{a}$ & $100 \mathrm{a}$ & $100 \mathrm{a}$ \\
\hline C.V. $(\%)$ & & 2,4 & 1,0 & 2,2 & 0,0 & 0,0 & 0,0 \\
\hline
\end{tabular}

1 Para análise, os dados foram transformados em arco seno de raiz de $\mathrm{x} / 100$.

2 DAA= dias após a aplicação dos produtos

3 Médias seguidas pela mesma letra na coluna não diferem entre si pelo teste de Tukey a 5\% de probabilidade.

Tabela 5. Percentagem de controle do caruru-de-mancha (Amaranthus viridis L. - AMAVI) nas avaliações realizadas aos 14,28 e 42 dias após a aplicação. Paulínia, SP.

\begin{tabular}{|c|c|c|c|c|}
\hline \multirow[t]{3}{*}{ Tratamento } & \multirow{3}{*}{$\begin{array}{c}\text { Dose } \\
\text { (kg/ha) }\end{array}$} & \multicolumn{3}{|c|}{ Percentagem de controle $^{1}$} \\
\hline & & \multicolumn{3}{|c|}{ AMAVI } \\
\hline & & 14 DAA $^{2}$ & 28DAA & 42DAA \\
\hline Test. sem capina & 0 & $0 b^{3}$ & $0 \mathrm{~b}$ & $0 \mathrm{~b}$ \\
\hline Test. capinada & 0 & $100 \mathrm{a}$ & $100 \mathrm{a}$ & $100 \mathrm{a}$ \\
\hline Isoxaflutole & 0,075 & $100 \mathrm{a}$ & $100 \mathrm{a}$ & $100 \mathrm{a}$ \\
\hline Isoxaflutole & 0,150 & $100 \mathrm{a}$ & $100 \mathrm{a}$ & $100 \mathrm{a}$ \\
\hline Isoxaflutole & 0,300 & $100 \mathrm{a}$ & $100 \mathrm{a}$ & $100 \mathrm{a}$ \\
\hline Metribuzin & 0,720 & $100 \mathrm{a}$ & $100 \mathrm{a}$ & $100 \mathrm{a}$ \\
\hline Metribuzin + linuron & $0,910+0,384$ & $100 \mathrm{a}$ & $100 \mathrm{a}$ & $100 \mathrm{a}$ \\
\hline C.V. $(\%)$ & & 0,0 & 0,0 & 0,0 \\
\hline
\end{tabular}

1 Para análise, os dados foram transformados em arco seno de raiz de $\mathrm{x} / 100$.

2 DAA= dias após a aplicação dos produtos

3. Médias seguidas pela mesma letra na coluna não diferem entre si pelo teste de Tukey a $5 \%$ de probabilidade. 
porcionou um controle de $98 \%$. Aos $42 \mathrm{DAA}$, todos os tratamentos controlaram igualmente a nabiça. Em relação ao controle do picão-branco e do caruru-de-mancha (Tabelas 4 e 5, respectivamente), verificou-se que em todos os tratamentos com herbicidas e em todas as épocas de avaliação, estes foram totalmente controlados. Adoryan et al. (2002) observaram que caruru-de-mancha foi controlado mesmo com a dose de $0,075 \mathrm{~kg} / \mathrm{ha}$.

Todos os tratamentos com herbicidas aumentaram a produção de tubérculos quando comparado com a testemunha onde não foi realizado controle de plantas daninhas (Tabela 6). Na testemunha capinada foi produzido $19,65 \mathrm{t} / \mathrm{ha}$ de

Tabela 6. Produção de tubérculos de batata expresso em t/ha. Paulínia, SP.

\begin{tabular}{lcc}
\hline Tratamento & $\begin{array}{c}\text { Dose } \\
\text { (kg/ha) }\end{array}$ & $\begin{array}{c}\text { Produção de } \\
\text { tubérculos } \\
\text { (t/ha) }\end{array}$ \\
\hline Test. sem capina & 0 & $6,30 \mathrm{~b}^{1}$ \\
Test. capinada & 0 & $19,65 \mathrm{a}$ \\
Isoxaflutole & 0,075 & $21,75 \mathrm{a}$ \\
Isoxaflutole & 0,150 & $24,75 \mathrm{a}$ \\
Isoxaflutole & 0,300 & $22,20 \mathrm{a}$ \\
Metribuzin & 0,720 & $25,50 \mathrm{a}$ \\
Metribuzin + linuron & $0,910+0,384$ & $20,60 \mathrm{a}$ \\
\hline C.V.(\%) & \multicolumn{3}{c}{13,7} \\
\hline 1. Médias seguidas pela mesma letra na coluna não diferem entre \\
\multicolumn{2}{c}{$\quad$ si pelo teste de Tukey a 5\% de probabilidade. }
\end{tabular}

tubérculos de batata enquanto que na testemunha sem controle apenas 6,3 t/ha mostrando que as plantas daninhas exerceram forte competição pelos fatores de produção, reduzindo em $70 \%$ a produção. A produção de tubérculos foi beneficiada pelo uso de herbicidas quando comparada com a testemunha sem capina. Não houve diferença na produção quando comparou-se os tratamentos com herbicidas e o tratamento capinado. Isoxaflutole em qualquer dose aplicada não afetou a produtividade dos tubérculos de batata.

\section{CONCLUSÕES}

1) Isoxaflutole foi seletivo à cultura da batata nas doses de $0,075,0,150$ e $0,300 \mathrm{~kg} / \mathrm{ha}$.

2) Todas as espécies daninhas avaliadas no experimento foram eficientemente controladas com isoxaflutole e com metribuzin.

3) Os tratamentos com isoxaflutole proporcionaram maior controle que aquele com metribuzin + linuron para o controle de Brachiaria plantaginea e Digitaria horizontalis.
4) controle de plantas daninhas beneficiou a produção de tubérculos, acarretando aumento de $70 \%$ na produção.

5) Não houve diferença entre os tratamentos com herbicidas e a testemunha capinada quanto à produção de batata.

\section{LITERATURA CITADA}

ADORYAN, M.L.; GELMINI, G.A.; VICTÓRIA FILHO, R. Controle de plantas daninhas na cultura da batata com isoxaflutole. In: CONGRESSO BRASILEIRO DA CIÊNCIA DAS PLANTAS DANINHAS, 23. Gramado, RS, 2002. Resumos... Londrina: SBCPD, 2002. p.538.

ANDREI. Compêndio de defensivos agrícolas. 6 ed. São Paulo: Andrei, 1999.672p.

GELMINI, G.A. ; CHRISTOFFOLETI, P.J. Herbicidas: indicações básicas para a cultura da batata. Campinas: CATI. 1997. 15p.

GIBSON, R. Pigment biosynthesis inhibitors. In: GIBSON, L.R. Principles of weed science: herbicide mode of action. 2001. Iowa State University. 13p. Disponível em: $<\underline{\text { http:/ }}$ /www.agron.iastate.edu/courses/agron317/ Pigment Inhibitors.htm>. Acesso em: 9 maio 2002. (Agronomy, 317).

GRANDO, W. Batata: principais pragas e doenças. Correio Agrícola Bayer, São Paulo, edição n.1, p.10-13, 2002.

GUNSOLUS, J.L. ; CURRAN, W.S. Herbicide mode of action and injury symptons. Revisado em 1999. St. Paul: University of Minnesota Extension Service. 20p. (North Central Regional Extension Publication, 377). Disponível em: <http://www.extension.umn.edu/distribution/ cropsystems/DC3832.html>. Acesso em: 9 mai. 2002.

HAGER, A. ; McGLAMERY, M. Corn injury from Balance herbicide. 1999. University of Illinois Extension. Disponível em:<http://www.ag.uiuc.edu/cespubs/pest/ articles/199910k.html>. Acesso em: 9 maio 2002.

MIRANDA FILHO, H.S. Batata. In: RAIJ, B. van; CANTARELLA, H.; QUAGGIO, J.A.; FURLANI, A.M.C. (eds.). Recomendações de adubação e calagem para o Estado de São Paulo. 2 ed. Campinas: Instituto Agronômico \& Fundação IAC, 1996. p.225. (Boletim técnico, 100).

MITRA, S.; BHOWMIK, P.C.; XING, B. Sorption of isoxaflutole by five different soils varying in physical and chemical properties. Pesticide Science, v.55, p.935-942, 1999. 
Márcio L. Adoryan et al.

PROSTKO, E.P. ; BAUGHMAN, T.A. (eds.) Peanut herbicide injury symptomology guide. Texas Agricultural Extension Service/The Texas A \& M University System. 1999. 11p.

REGEHR, D.L. New corn herbicides. In: Agronomy in action covering crops, soils, range, and weed science. Manhattan: Kansas State University/Cooperative Extension Service, 1998. p.2-3.

RODRIGUES, B.N. ; ALMEIDA, F.S. Guia de herbicidas. 4 ed. Londrina: Edição dos autores, 1998. 648p.

SPADER, V. ; VIDAL, R.A. Interferência de Brachiaria plantaginea sobre características agronômicas, componentes do rendimento e produtividade de grãos de milho. Planta Daninha, v.18, p.465-470, 2000.
VIEIRA, F.C. Manejo de plantas daninhas na cultura da batata. Piracicaba: ESALQ, 2000.

WICKS, G.A.; KLEIN, R.N.; WILSON, R.G.; ROETH, F.W.; KNEZEVIC, S.; MARTIN, R. Isoxaflutole (Balance) herbicide injury in Nebraska. University of Nebraska. [9-05-2002]. 8p. Disponível em: <http:// www.alfi.soils.wisc.edu/extension/FAPM/proceedings/ 4B.wicks.pdf>. Acesso em: 9 maio 2002.

YOUNG, B.G. ; HART, S.E. Optimizing foliar activity of isoxaflutole on giant foxtail (Setaria faberi) with various adjuvants. Weed Science, v.46, p.397-402, 1998. 